\title{
Effect of Systemic Administration of Simvastatin on Dental Implant Stability: A Random Clinical Study
}

\author{
Ali Mohammed Hassan, \\ B.D.S. = Master Student, Department of Oral and Maxillofacial Surgery, College of Dentistry, University of Baghdad. \\ Adil Al Kayat,
}

B.D.S., M.Sc. Sheffield, FDSRCS London. - Assistant Professor, Chairman of the Scientific Council of Oral and Maxillofacial Surgery, Iraqi Council of Medical Specialities.

\begin{abstract} implant placement (at surgery) and after 8,12 weeks respectively.

12 weeks in comparison to that of the control group. for both times $<0.001$ ) with the attributed risk percent was 70.8 and 50 respectively.

\section{KEYWORDS}

Implant Stability, Simvastatin, Resonance Frequency Analysis.

\section{INTRODUCTION}

The dental implant is increasingly becoming a popular treatment for replacing missing teeth for partially dentated as well as edentulous patients. In 2011 alone, dentists across the U.S. placed over five million implants, according to the American Dental Association ${ }^{(5)}$.
\end{abstract}

Background: the primary objective for many researches carried out in dental implantology was to reduce the period needed for functional implant loading, simvastatin (cholesterol lowering medication) had many pleiotropic effects, one of which was increasing bone density around titanium implants (1) and subsequently establishing faster osseointegrated dental implants $(2,3)$. Aim of the study: this study aims to reduce the period of time needed to establish secondary stability of dentalimplant measured in ISQ (Implant Stability Quotient) by investigating the effect of orally administered simvastatin on bone.

Materials and methods: simvastatin tablets $(40 \mathrm{mg} /$ day for three months) were administered orally for 11 healthy

womenaged (40-51) years old who received 15 dental implants (Dentium, Implantium) in the traumatic functionalimplant zone(4), this is the intervention group, the control group $(n=11)$ received 14 dental implants in the same zone.

3 dental implants in 2 subjects were lost, leaving a total of 26 dental implants in 20 patients with 10 patients in each

group. All subjects were radiographed with OPG for preliminary assessment and with CT scan for registering bone density in Hounsfield Units. Different dental implant sizes were used according to optimal patients' needs. an informed consent was obtained from the intervention group and the recommended monitoring protocol was followed.

Dental implant stability ISQ were recorded using RFA by Osste IIM ISQ for both groups three times: immediately after

Results: results showed that the mean implant stability for the intervention group was significantly higher $\mathrm{P}=0.01$ after

Simvastatin showed statistically significant effect on implant stability among the intervention group after 8 and 12 weeks (P value

Conclusions: this study concluded that the intervention group had higher implant stability and was ready forfunctional loading prior to control group and that simvastatin might enhanced and/or accelerated the process of osseointegration.

Osseointegration was first described by Brånemark and co-workers ${ }^{(6)}$. The term was first defined in a paper by Albrektsson et al 1981 as direct contact (at the light microscope level) between living bone and implant ${ }^{(7)}$. Since the histological definitions have some shortcomings, mainly that they have a limited clinical application, another more biomechanically oriented definition of osseointegration has been suggested: "A process whereby clinically asymptomatic rigid fixation of alloplastic materials is achieved, and maintained, in bone during functional loading" (8).

Over the following years attempts have been made by researchers to improve dental implant osseointegration (clinically applicable in terms of dental implant stability) through understanding the factors influencing it and the production of various materials in favor of that concept. One of these newly studied materials is Simvastatin.

Simvastatin is a 3-hydroxy-3-methylglutarylcoenzyme A (HMG-CoA) reductase inhibitor. It is widely used as a cholesterol-lowering drug and inhibits hepatic cholesterol biosynthesis. Recent studies have shown a beneficial effect of statins on bone mineral density (BMD) ${ }^{(9,10)}$. It has been suggested that several statin drugs, including simvastatin, increase the mRNA expression of bone morphogenetic protein (BMP-2) in osteoblasts, with a subsequent increase in bone formation. Simvastatin has been shown to enhance osseointegration of pure titanium implants in osteoporotic rats. ${ }^{(1)}$ Other experimental study shows that locally administered simvastatin was detrimental to the repair of defects in the calvaria of rats ${ }^{(12)}$. The period required generally for an osseointegration to be achieved and for dental implant to be loaded is about 3-6 months which still represent a relatively long period for patients and any efforts focusing on reducing this period are entitled for consideration and scientific research which is the objective of this study.

\section{MATERIALS AND METHOD}

This study was conducted at the dental implant unit in Oral and Maxillofacial Department of College of Dentistry, Baghdad University, from January 2012 to February 2014, where twenty two healthy females aged (40-51) years old received 29 dental implants (Dentium, Implantium) were divided randomly (using alternating randomization method) into two groups, control and intervention group. 3 dental implants in 2 subjects were lost, leaving a total of 26 dental 
implants in 20 patients with 10 patients in each group:

The intervention group, this group received 14 titanium screw type endosseous dental implants (Dentium, Implantium) in the traumatic functional implant zone (the area from maxillary right 1st premolar to the maxillary left 1st premolar) along with systemically administrated (oral) simvastatin $40 \mathrm{mg} /$ day (as an accepted dose for humans) ${ }^{(13)}$ postoperatively for three months.

The control group, this group received 12 titanium screw type endosseous dental implants (Dentium, Korea) in the traumatic functional implant zone and submitted to the same procedure of intervention group without the administration of post-operative simvastatin.

\section{Exclusion Criteria:}

\section{a.Smokers.}

b.Alcoholics.

c.Patients with any chronic systemic disease. For example (active liver disease, patients on warfarin and/or antifungal medication and/or cyclosporine... etc.)

d.Pregnant or lactating females.

e.Patients with inadequate sub-antral distance, due to maxillary sinus neumatization ${ }^{(14)}$.

f.Implant site subjected previously to supplemental surgical procedures (bone graft, ridge augmentation.... etc.).

The prospective implant sites were examined clinically and radiographically by two views: Orthopantomogram (OPG) and CT scan for registering bone density (using Hounsfield units and according to Misch classification of bone quality) (15) at the target site and also for precise placement of the dental implants through providing information about width of the alveolar bone and proximity to the maxillary sinus. After the patients signed an informed consent expressing their approval for participating in this study the insertion of fixtures is carried out in the traumatic zone for both groups then the primary stability is measured immediately after dental implant placement by a colleague for each patient by Resonance Frequency Analysis (RFA) using OsstellTM ISQ (Goteborg, Sweden) through inserting the smart peg into the implant and two readings of the ISQ (Implant Stability Quotient) values are recorded; in bucco-palatal direction and the other in mesiodistal one.

Implant stability was measured again after 8 and 12 weeks postoperatively by the same colleague using RFA to compare its values (ISQs) between both groups.

Group A were asked to perform a liver function test (SGPT, SGOT) after 6 and 12 weeks postoperatively as a monitoring for any hepatic side effects and to report any muscular/joint pain, spasm or discomfort for further CPK (Creatine Phosphokinase) evaluation (16).

\section{RESULTS}

\section{Statistical Analysis}

Statistical analyses were done using SPSS version 21 computer software (Statistical Package for Social Sciences) in association with Microsoft Excel 2010. In this study the following statistics were used: 1.Descriptive Statistics: including; mean, standard deviation and standard error.

2.Parametric statistical tests of significance: including; t-test, Paired t-test and Cohen's d (standardized measure of effect size).

\section{The Difference in Mean Stability between the Two Groups}

(Table 1) shows a comparison of dental implant mean stability between both groups through time intervals, where in group A after 8 weeks of surgery there was an increase in the mean stability which was not statistically significant by 1.8 ISQ units compared to the primary stability readings. The changes observed during the 1st 8 weeks of surgery were evaluated as a weak effect (Cohen's $\mathrm{D}=0.22$ ).

Table 1: Mean-ISQ of the 2 perpendicular directions at surgery and after 8 weeks.

\begin{tabular}{|c|c|c|c|c|c|}
\hline Control & at surgery & After 8 weeks & Changs compared to baseline & Cohen's $\boldsymbol{d}$ & P (Paired t-test) \\
\hline N & 12 & 12 & 12 & & \\
\hline Mean & 72.8 & 66 & -6.7 & -0.65 & 0.045 \\
\hline SD & 8.2 & 6.3 & 10.3 & & \\
\hline SE & 2.36 & 1.81 & 2.97 & & \\
\hline Intervetion & & & & & \\
\hline N & 14 & 14 & 14 & 0.22 & $0.44[\mathrm{~N} \mathrm{~S}]$ \\
\hline Mean & 73.5 & 75.3 & 1.8 & & \\
\hline SD & 7 & 6.6 & 8.3 & & \\
\hline SE & 1.87 & 1.78 & 2.21 & \\
\hline
\end{tabular}


(Table-2) Mean-ISQ of the 2 perpendicular directions after 12 weeks and compared to primary stability

\begin{tabular}{|c|c|c|c|c|}
\hline & after $\mathbf{1 2}$ weeks & changes compared to baseline & Cohen's $\boldsymbol{P}$ & P (paired t-test) \\
\hline Control & & & & \\
\hline N & 12 & 12 & -0.42 & $0.17[\mathrm{NS}]$ \\
\hline Mean & 69.3 & -3.5 & & \\
\hline SD & 5 & 8.3 & & \\
\hline SE & 1.44 & 2.4 & & \\
\hline Intervention & & & & \\
\hline N & 14 & 14 & & \\
\hline Mean & 79.5 & 5.9 & \\
\hline SD & 3.9 & 7.3 & \\
\hline SE & 1.05 & 1.96 & \\
\hline
\end{tabular}

The total change in mean ISQ after 12 weeks of the surgery compared to the primary stability was an average increase of 5.9 ISQ units which is statistically significant $(\mathrm{P}$ value $=0.01)$, and the effect evaluated as a strong effect (Cohen's $\mathrm{D}=0.81$ ).

The whole behavior of implant stability for both groups is shown in (figure 1)

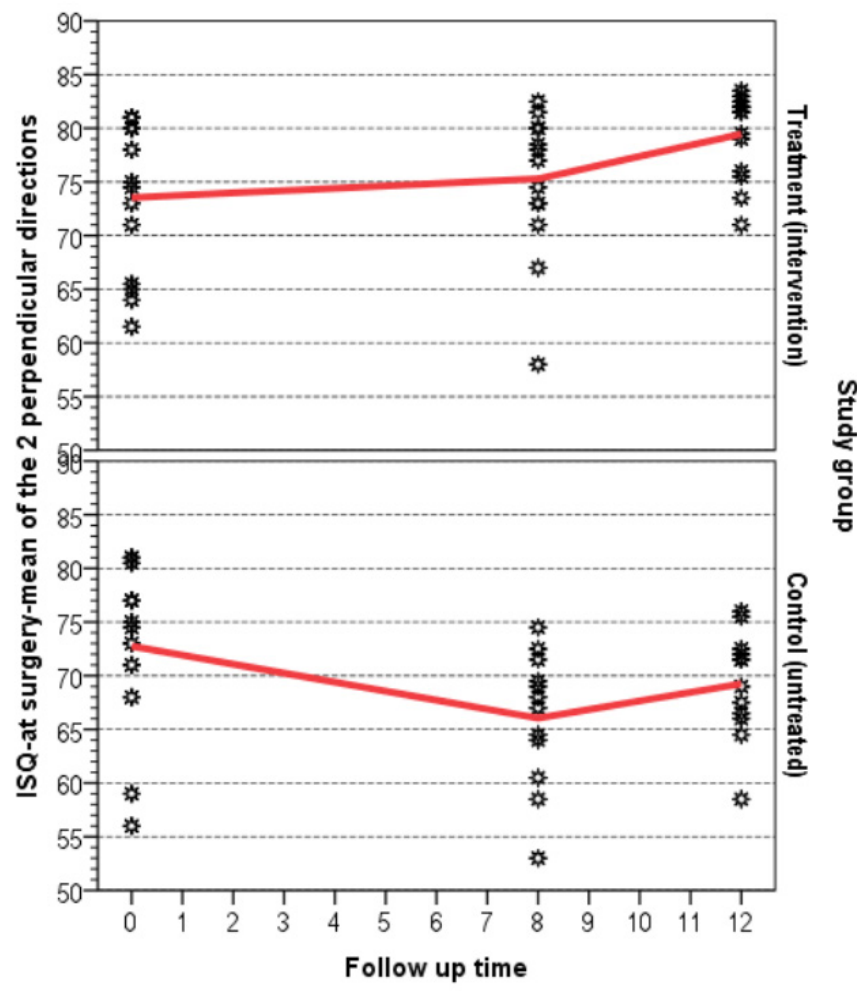

Figure 1: illustrating the mean stability of the dental implants for both control and intervention groups where the left (Y) axis represent the ISQ units and the horizontal

(x) axis represent the time measured in weeks.

\section{Number of Implants achieved 70+ ISQ in Both Groups}

The stability of implant at 70 ISQ or more is considered an implant with high ISQ stability (17), statistical test using attribute risk percent was used to compare numbers of dental implants in treated versus non treated control group that achieved the bench mark of implant stability (70+ ISQ) over time as illustrated in (figure 2) below.

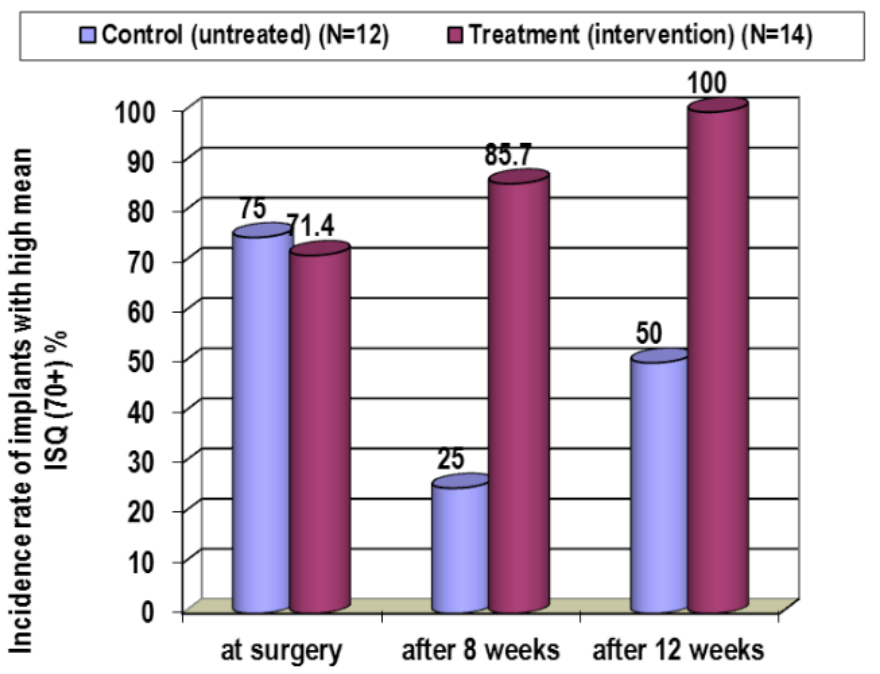

Figure 2: number of implants achieved high stability

In (figure 2) the control group and the intervention group show no difference at surgery but after 8 weeks $25 \%$ of the control group reached the high ISQ level while in the intervention group $85.7 \%$ achieved the high stability level of 70+ ISQ.

At week 12, 50\% of the control group dental implants reached to the bench mark level of high implant stability in comparison to the intervention group where $100 \%$ of the dental implants were at high implant stability (all the dental implants in the intervention achieved 70+ ISQ at the end of the study).

\section{DISCUSSION}

To the best of the authors' knowledge; this study is the first and no other comparable studies were available. The sample selection was based on two basic features: gender and age. Females were selected over males because the changes in bone remodeling occur in a faster rhythm, And their age was $40+$ years old and not younger because in this range of age most of bone parameters regarding bone regeneration capacity, elasticity, strength and even cell viability 
are declining especially in females aged above 40 years old, where $34 \%$ had osteopenia and $8 \%$ had osteoporosis ${ }^{(18)}$.

Monitoring protocol was applied throughout this study to all intervention group, their liver function tests were negative and within the normal range $($ SGPT $<34 \mu / 1$, SGOT $<31 \mu / 1)$.

Members of the intervention group were asked routinely to report any muscular pain, cramps, and weakness. No such reports or complaints were informed.

Among the control group; the mean ISQ after 8 weeks of surgery showed an obvious reduction which re-increased to an obvious increment till 12 weeks. The overall change after 12 weeks compared to primary stability at surgery was still in the negative direction making the final stability still less than the primary one; yet they are loadable. This pattern is well documented in literature especially for mean primary stability of more than 70 ISQ giving the fact that the conventional 2-stage treatment loading protocol used a period of 3-6 months for osseointegration prior to loading ${ }^{(19,20)}$. However, Simvastatin treatment changed the pattern described earlier for the control group. The mean ISQ for the intervention group was obviously higher compared to primary stability (mean ISQ at surgery was 73.5 and at the 8th week was75.3), although the positive changes observed were less than the level of statistical significance; nevertheless, it contradicted the negative trend observed for ISQ in the first 8 weeks in untreated control group (mean ISQ at surgery was 72.8 and at the $8^{\text {th }}$ week was 66 ).

This positive trend in ISQ change in treatment group continued till the end of the study, making the stability after 12 weeks significantly and noticeably higher than that of primary stability. This finding is opposite to the negative loss in ISQ in relation to primary stability observed in control group within the scope and parameters of the current study.

Another advantage for effectiveness of simvastatin on dental implant stability was the absence of difference in mean ISQ at primary stability between the two groups (control 72.8 ISQ, intervention 73.5 ISQ, the difference was 0.7 ISQ), while after 8 and 12 weeks the intervention group had a significant advantage around 10 ISQ units increase in mean difference over the untreated control group (control group mean ISQ at week 8 and 12 was 66, 69.3 respectively while intervention group mean ISQ at week 8 and 12 was $75.3,79.5$ respectively). Since these numbers represent the mean stability, it doesn't mean that each individual dental implant of the intervention group is necessarily ready for immediate loading.

To summarize the current study outcome, it is worth mentioning that an almost comparable proportion of subjects had high primary stability $(>70$ ISQ) in both control $(75 \%)$ and simvastatin treated group (71.4\%). After 8 weeks of surgery the rate of high stability in the intervention group increased to (85.7\%) while among those untreated (control group) only $(25 \%)$ had high stability.

After 12 weeks, all simvastatin treated group achieved the bench mark of implant stability (70+ ISQ) while only $50 \%$ of untreated group achieved this favorable outcome which they may reach it eventually on the expense of time.

In conclusion, Simvastatin administration had reduced the generally needed functional loading time in traumatic functional implant zone of dental implants from 3-6 months (12-26 weeks) to almost 2 months ( 8 weeks) by enhancing osseointegration of dental implant and increasing its stability faster than that in control group. Simvastatin was well tolerated in all healthy subjects as they were submitted for periodic monitoring (liver function test) and all tests were normal and no subject reported muscular pain or weakness.

Further recommendation is to Measure dental implant stability at shorter time intervals (after 7 weeks from primary stability) to detect earlier changes associated with the drug. Inclusion of a larger sample for more conclusive results. A longer period of follow up to evaluate the long term effect on success rate of dental implants.

\section{REFERENCES}

1. Du Z, Chen J, Yan F, Xiao Y. Effects of Simvastatin on bone healing around titanium implants in osteoporotic rats. Clinical oral implants research 2009;20(2):145-150.

2. YangF,ZhaoS.F,ZhangF,HeF.M,YangG.L. Simvastatinloaded porous implant surfaces stimulate preosteoblasts differentiation: an in vitro study. Oral Surgery, Oral Medicine, Oral Pathology, Oral Radiology, and Endodontology 2001;111(5): 551-556.

3. Nyan M, Hao J, Miyahara T, Noritake K, Rodriguez R, Kasugai S. Accelerated and Enhanced Bone Formation on Novel Simvastatin-Loaded Porous Titanium Oxide Surfaces. Clinical implant dentistry and related research 2013.

4. Tolstunov L. Implant zones of the jaw: Implant location and related success rate. J Oral Impl 2007; 33(4), 211-20.

5. http://www. American dental association.org.2014, January.

6. Branemark P, Hansson B, Adell R, Breine U, Lindstrom J, Hallan O, Ohman A. Osseointegrated implants in the treatment of the edentulous jaw. Experience from a 10years period. Scand J Plast Reconstr Surg Suppl 1977; 16, $1-132$.

7. Albrektsson T, Brånemark PI, Hansson HA, Lundström 
I. Osseointegrated titanium implants. Requirements for ensuring a long-lasting, direct bone- to-implant anchorage in man. Acta Orthop Scand. 1981;52(2):155-70.

8. Mantri, S., Khan, Z. Prosthodontic Rehabilitation of Acquired Maxillofacial Defects. Head and Neck Cancer. Intech, 2012; 315-36.

9. Klçcoglu S. S., Erdemli E. New addition to the statin's effect. Journal of Trauma-Injury, Infection, and Critical Care 2007; 63(1), 187-191.

10. Uzzan B, Cohen R, Nicolas P, Cucherat M, Perret G.Y. Effects of statins on bone mineral density: a meta-analysis of clinical studies. Bone, 2007; 40(6), 1581-1587.

11. Du Z, Chen J, Yan F, Doan N, Ivanovski S, Xiao Y. Serum bone formation marker correlation with improved osseointegration in osteoporotic rats treated with simvastatin. Clin Oral Imp Res. 2011

12. Calixto J, Villaboim de Castro Lima C, Frederico L, Pio dos Santos de Castro Lima R, Anbinder A. The influence of local administration of simvastatin in calvarial bone healing in rats. Journal of Cranio- Maxillofacial Surgery, 2011; 39(3), 215-220.

13. Park JB. The use of simvastatin in bone regeneration. Med Oral Patho 2009; 14(9), 485-8.
14. Calvo L, Gómez-Moreno G, López-Marí L, Ortiz-Ruiz A.J, Guardia-Muñoz J. Atraumatic maxillary sinus elevation using threaded bone dilators for immediate implants. A three-year clinical study. Med Oral Patol Oral Cir Bucal, 2010; 15(2), e366-70.

15. Misch C. Contemporary implant dentistry. St Louis, Missouri: Mosby, Elsevier. 3rd edi. 2008; 137.

16. Phillip O, James E, William G. Handbook of clinical drug data. New York: McGraw-Hill Publications, 10 edi, 2002.

17. Sennerby L. 20 years of experience with the resonance frequency analysis. Implantologie 2013;21(1):21-33.

18. Unni J, Grag R, Pawar R. Bone mineral density in women above 40 years. J Midlife Health 2010; 1(1), 19-22.

19. Atieh M, Alsabeeha N, Duncan W, Silva R, Cullinan M, Schwass D, Payne A. Immediate single implant restorations in mandibular molar extraction sockets: a controlled clinical trial. Clinical oral implants research, 2013; 24(5), 484-496.

20. Funato A, Yamada M, Ogawa T. Success rate, healing time and implant stability of photofunctionalized dental implants. Int J Oral Maxillofac Imp 2013; 28, 1261-71. 